\title{
Repair of a complex incisional hernia in the setting of palliative care
}

\author{
Clare O'Connell, Orla Mc Cormack, Faisal Awan, Naraynasamy Ravi, John V. Reynolds \\ Department of Surgery, St. James's Hospital, Dublin, and Trinity College Dublin, Ireland \\ Correspondence: Orla Mc Cormack. Address: Department of Surgery, St. James's Hospital, James's St. Dublin 8, \\ Ireland. Telephone: 003-531- 410-3000. Email: omccormack@rcsi.ie.
}

Received: June 25, 2012

Accepted: October 26, 2012

Online Published: March 6, 2013

DOI : $10.5430 / j b g c . v 3 n 2 p 73$

URL: http://dx.doi.org/10.5430/jbgc.v3n2p73

\section{Abstract}

Palliative surgery for cancer is mainly related to the palliation of direct or indirect causes of incurable disease. Surgery may also have to considered in patients with incurable disease for benign conditions that present significant symptoms or quality of life threats.

We present here in one such case of the radiological investigation and management of a large incisional hernia at the site of a previous appendicectomy incision in a patient with terminal non-small cell lung cancer (NSCLC) and multiple comorbidities.

A significant improved quality of life was gained by this surgery despite it being high risk.

\section{Key words}

Incisional hernia, Palliative surgery, Lung cancer

\section{Introduction}

Incisional herniae are relatively common sequelae of abdominal surgery ${ }^{[1]}$. They can occur early or many years after surgery. The predisposing factors for development of an incisional hernia are wound infections, immunosuppression, diabetes, obesity, smoking, chronic cough, malnutrition, pregnancy, constipation, dialysis and excess fluid retention ${ }^{[2]}$. Not all herniae require repair, but symptomatic herniae, in particular painful herniae, and those complicated by intestinal obstruction or strangulation require surgery. Radiological investigations such as ultrasound and computed tomography (CT) are invaluable in aiding decision making. Advances in anaesthesia permit hernia surgery even in patients with considerable co-morbidities. The surgery of symptomatic herniae is rarely described in the context of palliative care in cancer, and we report herein one such case in which surgery was undertaken with a successful outcome.

\section{Case presentation}

A 72 year old man was diagnosed in 2009 with a non small cell lung cancer (NSCLC) and was staged as T1a N3. He had multiple co-morbidities including chronic obstructive pulmonary disease (COPD), diabetes mellitus type II, hypertension, 
hypercholesterolaemia and obesity. In his past surgical history, he had undergone an open appendicectomy fifty years previously.

He was treated with chemotherapy and radiotherapy. On follow up imaging, he had developed a right sided renal cell carcinoma and was subsequently treated with radio-frequency ablation. At the time he was noted to have a large incisional hernia through the site of the previous appendicectomy incision.

In late 2011 he was admitted as an emergency with increasing shortness of breath, progressive disease was diagnosed and he was reviewed by the palliative care service. He complained bitterly with severe lower abdominal pain and pressure, and on assessment his incisional hernia was irreducible. He was unable to walk comfortably and as a result it was impacting on his activities of daily living. On examination there was a $20 \times 18 \mathrm{~cm}$ visible hernia and the overlying skin was stretched and ulcerated (see Figure 1, Figure 2). CT imaging showed a wide-necked hernia containing loops of small bowel and colon in the hernia sac (see Figure 3, Figure 4).

Figure 1. Large incisional hernia

Photograph of large right sided abdominal incisional hernia

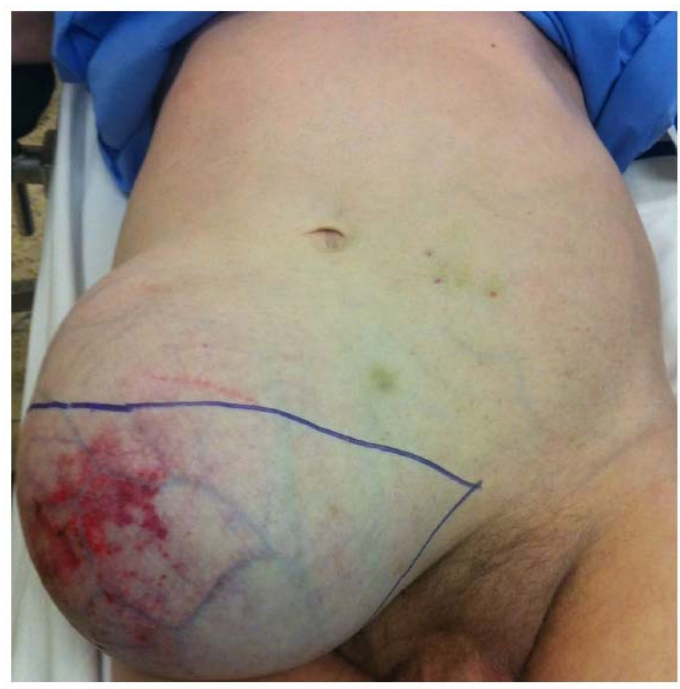

Figure 2. Lateral view of incisional hernia

Note the skin ulceration

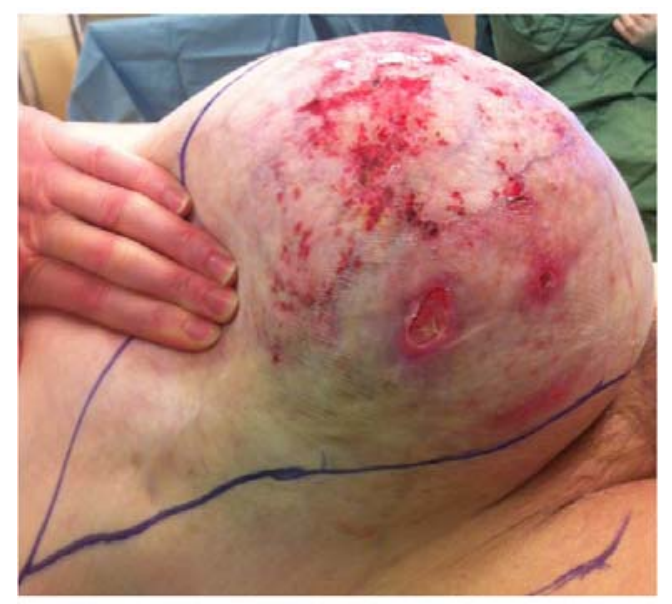

This patient had an extensive list of co morbidities that posed a huge operative and anaesthetic risk. He also had a progressive terminal illness. Moreover, he was in distress with pain and discomfort from an irreducible hernia. Having discussed the options with the patient and his family, a decision was made to operate on him. 
Figure 3. CT Abdomen and pelvis

Coronal view showing a large right incisional hernia with small bowel and colon in the hernia sac

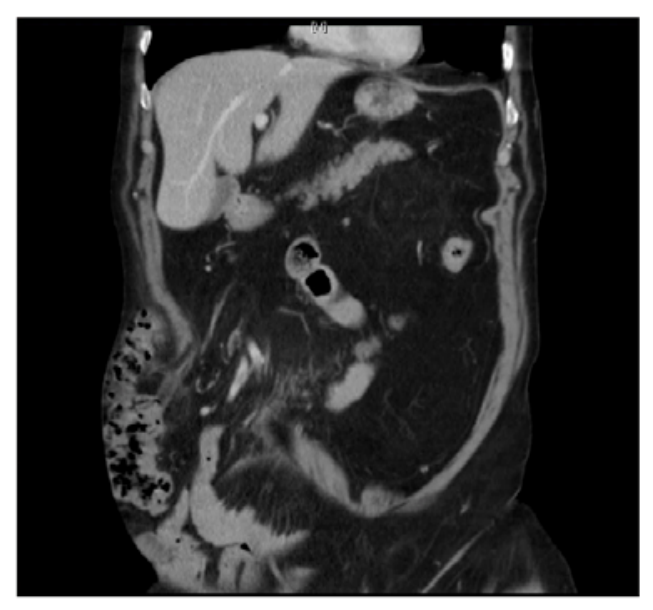

Figure 4. CT Abdomen and pelvis

Axial section of CT again showing small and large bowel contained in the hernia

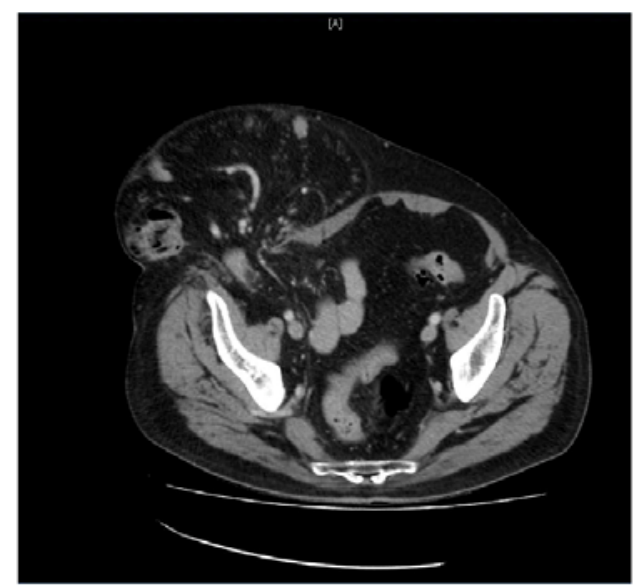

A spinal anaesthetic was administered. The contents of the hernia were reduced (see Figure 5), and the defect repaired with interrupted sutures and an on-lay polypropylene mesh (see Figure 6). The patient had an initial uneventful recovery and was discharged home well. Over the following two months, the patient lived at home and did well. He was then admitted to his local hospice for respite care and symptom control. Three months post-operatively he developed brain metastases, underwent a sudden rapid clinical deterioration and died.

Figure 5. Contents of hernia sac

Small bowel and its mesentery

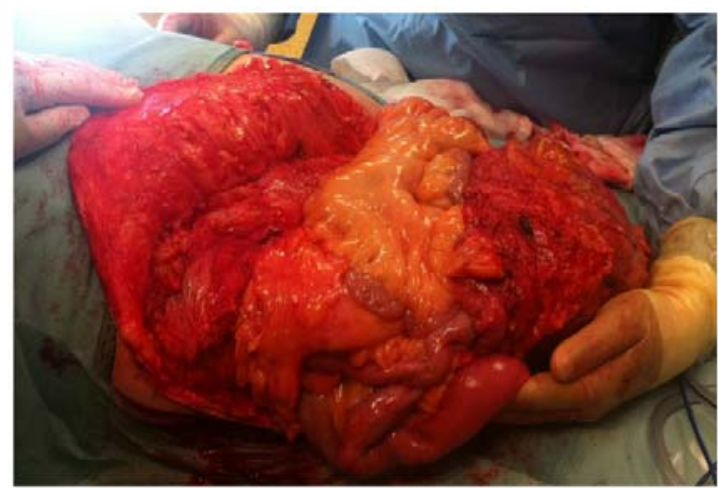


Figure 6. Intraoperative photograph

Polypropylene mesh sutured over repaired defect prior to closure of the skin

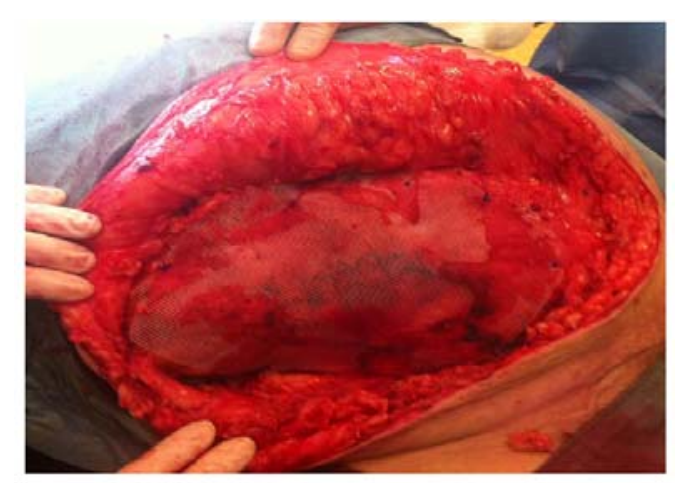

\section{Discussion}

There is a lack of consensus on the definition of the term 'palliative surgery' in the literature. Easson et al. propose that the purpose of palliative surgery is to relieve symptoms and suffering, but not to prolong life ${ }^{[3]}$. Potential benefits of surgery in palliative care are improvement in quality of life, pain reduction and increase in comfort, which must be balanced with the potential risk of surgical complications, which could shorten the patient's life further. Surgery in palliative care is invariably complicated by the underlying disease of the patient, and usually focused on complications caused directly or indirectly by the cancer. There has been an increased focus on the role of the surgeon in end of life care in the literature, with a shift from mortality and morbidity related outcomes to quality-of-life based outcomes ${ }^{[4]}$.

Miner et al analysed 1022 palliative surgical procedures performed in the setting of advanced cancer. Surgery was performed to relieve the symptoms of gastrointestinal obstruction (34\%), neurological symptoms (23\%), pain (12\%), dyspnoea (9\%), jaundice (7\%) or other symptoms (15\%). Median duration of symptom control was 135 and median survival after surgery was 194 days ${ }^{[5]}$.

Not all authors agree on surgical intervention in the palliative setting. Badgwell et al found in their study that $40 \%$ of all inpatient surgical oncology evaluations were requested for symptom palliation in patients with advanced or incurable malignancy. Their perioperative mortality (90-day) rate was 7\%, and they mention considerable morbidity rates. They conclude that although surgery can be performed with acceptable mortality in highly selected patients there may be considerable morbidity, thus they propose that non-operative management is more desirable in this cohort of patients ${ }^{[6]}$.

A more recent study, focussing on quality of life in patients with advanced malignancy evaluated for palliative surgical intervention, found that palliative surgical procedures constituted up to $13 \%$ of all operations performed by surgeons, with more than 1000 palliative procedures per year performed at major US cancer centres. In this study, only 1 of the 77 patients included, had surgery for a non cancer related symptom They concluded that although symptom resolution was achieved in $91 \%$ of patients, accurate quality of life assessment was difficult due to the early death rate in this population ${ }^{[7]}$.

We highlight this case as it is unusual, in that issues of palliation presented themselves in a patient with end-stage disease, as well as multiple co-morbidities, yet the greatest morbidity for the patient was unrelated to cancer. The goal of this operation was to alleviate pain and discomfort to allow the patient to enjoy a better quality of life, and this was achieved to the great satisfaction of the patient for the remainder of his life.

\section{References}

[1] Santora TA, Roslyn JJ. Incisional hernia. Surg Clin North Am. 1993 Jun; 73(3): 557-70. PMid:8497803 
[2] Sørensen LT, Hemmingsen UB, Kirkeby LT, Kallehave F, Jørgensen LN. Smoking is a risk factor for incisional hernia. Arch Surg. 2005 Feb; 140(2): 119-23. PMid:15723991 http://dx.doi.org/10.1001/archsurg.140.2.119

[3] Easson A, Asch M, Swallow CJ: Palliative general surgical procedures. In: The Surgeon and Palliative Care. Edited by Dunn GP. Surg Clin North Am. 2001; 10: 161-184.

[4] Dunn, GP. Surgical Palliation in Advanced Disease: Recent Developments. Current Oncology Reports. 2002 ; 4: $233-241$. http://dx.doi.org/10.1007/s11912-002-0021-3

[5] Miner TJ, Brennan MF and Jaques DP. A Prospective, Symptom Related, Outcomes Analysis of 1022 Palliative Procedures for Advanced Cancer. Ann Surg. 2004 Oct; 240(4): 719-26. PMid:15383799

[6] Badgwell BD, Smith K, Liu P, Bruera E, Curley SA, Cormier JN. Indicators of surgery and survival in oncology inpatients requiring surgical evaluation for palliation. Support Care Cancer. 2009 Jun; 17(6): 727-34. PMid:19083026 http://dx.doi.org/10.1007/s00520-008-0554-6

[7] Badgwell B, Krouse R, Cormier J, Guevara C, Klimberg S, Ferrell B. Frequent and Early death Limits Quality of Life in Patients with Advanced Malignancies Evaluated for Palliative Surgical intervention. Ann Surg Oncol. 2012 Nov; 19(12): 3651-8. PMid:22669450 http://dx.doi.org/10.1245/s10434-012-2420-5 